\title{
Application of Kissinger analysis to glass transition and study of thermal degradation kinetics of phenolic-acrylic IPNs
}

\author{
S GOSWAMI* and K KIRAN \\ Department of Polymer Engineering, Birla Institute of Technology, Ranchi 835 215, India
}

MS received 17 March 2011

\begin{abstract}
Degradation kinetics of sequential IPNs, based on novolac resin and poly (2-ethyl hexyl acrylate), are studied at linear heating rates of $2^{\circ} \mathrm{C} / \mathrm{min}, 5^{\circ} \mathrm{C} / \mathrm{min}, 10^{\circ} \mathrm{C} / \mathrm{min}$ and $20^{\circ} \mathrm{C} / \mathrm{min}$ by thermogravimetric analyser (TGA). Activation energy $\left(E_{\mathrm{a}}\right)$ and order $(n)$ of thermal decomposition reaction for IPNs and pure phenolic resin are evaluated from TGA curves using differential method of Freeman and Carroll. Decrease in $T_{\mathrm{g}}$ with an increase of acrylate content in IPNs are seen. Lower activation energy $\left(E_{\mathbf{a}^{\prime}}\right)$, as calculated by applying Kissinger equation, for the concerned transition of IPNs, compared to that of pure phenolic resin is quite evident from DSC study.
\end{abstract}

Keywords. Interpenetrating network; Freeman-Carroll method; activation energy; differential scanning calorimetry; Kissinger equation.

\section{Introduction}

Phenolic resins were the first commercially available polymeric material having wide industrial applications as paints, adhesives, coupling agents and various moulding materials (Gardziella et al 2000; Wolfgang 2002). This is mainly because of its excellent mechanical and thermal properties (Knop and Scheib 1979; Papa and Sunshine 1973). Mixing of polymers to get blend, interpenetrating network, composite etc considering phenolic resin as one of the components are widely used techniques of enhancement of physical and thermal properties (Wang et al 2002). The characteristics of interpenetrating network based on phenolic resin and acrylics has been studied extensively (Bandyopadhyay et al 2003). However, thermal behaviour of such system has not been explored quantitatively. Earlier studies on phenolic-acrylic IPNs showed lowering in glass transition temperature of IPNs indicating enhancement of toughness for IPNs but poor thermal stability compared to that of pure phenolic resin. The reason of such a drop in thermal stability of IPNs was anticipated from plausible chemical reactions between the component resins at elevated temperature (Chakrabarty et al 2005). Toughening of phenolic-acrylic IPNs was reported on the basis of lowering in $T_{\mathrm{g}}$ values for them with respect to pure phenol formaldehyde. This result was further supported by the possible curing reaction of novolac resin in presence of acrylic polymer. However, quantitative evaluation of activation energy and order of reaction, for the thermal degradation as well as thermal transition of phenolic-acrylic IPNs, is required to correlate the basis of the proposed chemical reaction with the thermal behaviour.

*Author for correspondence (sdptgoswami@yahoo.com; sdptgoswami@bitmesra.ac.in)
Certain kinetic models have been successfully developed and applied to evaluate the activation energy for the respective thermal degradation of the phenolic resin. The decomposition kinetics of phenolic resin (both novolac and resol type) under inert atmosphere, were studied by Hwang et al (2003) using the standard kinetic equation e.g. Flynn-wall expression and were correlated to different phenol/formaldehyde (P/F) molar ratios. Furthermore, Chang et al (2004) reported many interesting structural changes of phenolic resin during heat treatment in inert atmosphere, both in presence of $\mathrm{CO}_{2}$ and $\mathrm{N}_{2}$.

The present work involves preparation of sequential interpenetrating networks based on novolac resin and poly(2-ethyl hexyl acrylate), taken in varying weight ratios. Kissinger analysis is applied to analyse DSC thermograms of IPNs, evaluate activation energy for the glass transition and correlate the effect to the toughening of the phenolic matrix upon IPN formation. Relative thermal stability and the kinetics of degradation of IPNs, with respect to varying blend ratio $(w / w)$ has been studied to estimate the activation energy $\left(E_{\mathrm{a}}\right)$ and order of degradation reaction $(n)$ by using Freeman-Carroll method of analysis.

\section{Experimental}

\subsection{Materials}

Novolac, the precursor of the crosslinked phenolic resin mixed with $10 \%$ (of the novolac resin weight) of hexamethylene tetramnie (HEXA), was procured from Hindustan Adhesives (Kolkata, India) and used without further modification. Monomer (2-ethyl hexyl acrylate) (EHA) (Sigma Aldrich, Germany) was purified by washing first with equal 
volume of $2 \% \mathrm{KOH}$ solution in a separating funnel, and then thorough and repeated washings with distilled water to make it alkali free, as tested by litmus paper. Then it was dried over anhydrous calcium chloride $\left(\mathrm{CaCl}_{2}\right)$ for $3 \mathrm{~h}$ and finally filtered. The purified monomer was stored in the refrigerator. Benzoyl peroxide $\left(\mathrm{Bz}_{2} \mathrm{O}_{2}\right)$ from $\mathrm{CDH}$, India, was purified by repeated crystallization from chloroform. Ethylene glycol dimethacrylate (EGDMA) (Sigma Aldrich, Germany), without any modification, was used as co-monomer and crosslinker for poly (2-ethyl hexyl acrylate), PEHA.

\subsection{IPN synthesis}

Purified monomer 2-ethyl hexyl acrylate was mixed with $2 \%$ by weight (based on monomer) of $\mathrm{Bz}_{2} \mathrm{O}_{2}$. Novolac resin, premixed with HEXA, was mixed in a suitable weight ratio with acrylic monomer thoroughly and uniformly. The resulting mass was allowed to mature for $2 \mathrm{~h}$. The paste was then transferred into a positive type compression sheet mould $(10 \times 6 \times 3 \mathrm{~cm})$, which was preheated to $80^{\circ} \mathrm{C}$. The press was then closed with a mild pressure to keep the mould airtight and to ensure that no air was entrapped into the sheet. This condition was maintained for $30 \mathrm{~min}$ to allow the acrylic polymerization to initiate and propagate to a certain extent. Once the stipulated time period for the acrylic polymerization was over, the temperature of mould was increased to $150^{\circ} \mathrm{C}$ with simultaneous increase in pressure to about 5 tons $/ \mathrm{cm}^{2}$. The mould was kept under same condition for a few min to ensure completion of crosslinking of the phenolic resin and polymerization of acrylic as well. It was then removed from the press in hot condition and opened cautiously to avoid any distortion to the sheet. Samples for testing were cut from the sheet after maturing for 7 days. In case of full IPNs, the comonomer crosslinker was mixed up with liquid acrylate ( $2 \%$ by weight with respect to the monomer) prior to addition of it to Novolac powder. All other steps remained unaltered.

\section{Characterization}

\subsection{Differential scanning calorimetry (DSC)}

Thermal studies of IPNs were carried out using differential scanning calorimeter (TA instruments, USA; Q10) at 4 different heating rates $\left(2^{\circ} \mathrm{C} / \mathrm{min}, 5^{\circ} \mathrm{C} / \mathrm{min}, 10^{\circ} \mathrm{C} / \mathrm{min}\right.$ and $20^{\circ} \mathrm{C} / \mathrm{min}$ ), under continuous flow of nitrogen in a sealed aluminum pan starting from $20-220^{\circ} \mathrm{C}$. Initial sample weight ranged from $1.29-1.80 \mathrm{mg}$. Indium was used as standard to calibrate the temperature and heat flow.

\subsection{Thermogravimetric analysis (TGA)}

Phenolic resin-PEHA IPNs (semi and full) were subjected to thermogravimetric analyser (Shimadzu, Japan; DTG-60) under continuous flow of nitrogen gas at a constant heating rate of $10^{\circ} \mathrm{C} / \mathrm{min}$ in the range $30-800^{\circ} \mathrm{C}$. Samples ranging from 4.99-8.01 mg of weight were taken in aluminum crucible for analysis.

\section{Results and discussion}

\subsection{Differential scanning calorimetry}

Figure 1 shows DSC thermograms of semi-IPNs obtained at $10^{\circ} \mathrm{C} / \mathrm{min}$ heating rate. IPNs, with higher acrylate content, show lower glass transition temperature, $T_{\mathrm{g}}$, as determined

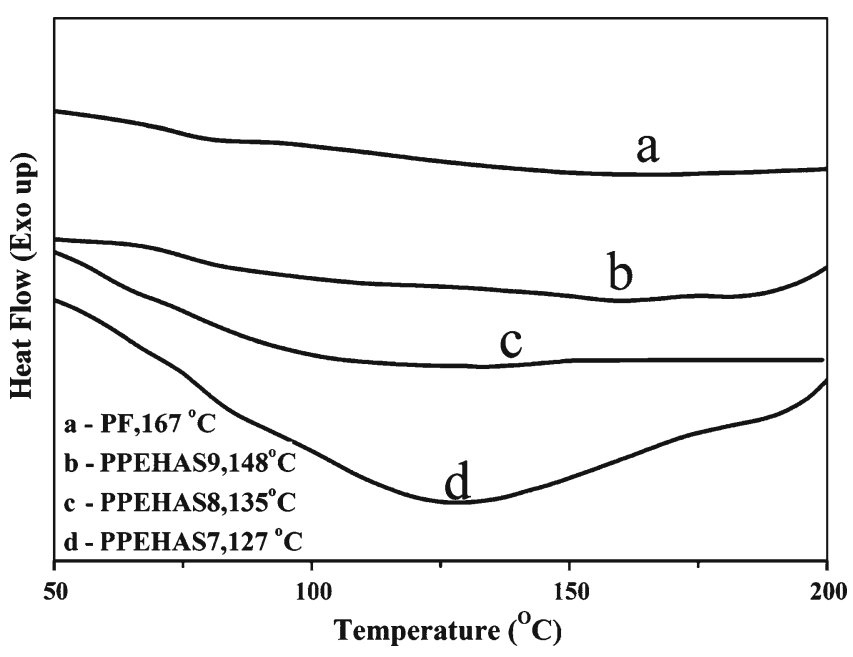

Figure 1. DSC thermograms of $\mathrm{PF}$ and PF/PEHA semi IPNs at heating rate of $10^{\circ} \mathrm{C} / \mathrm{min}$.

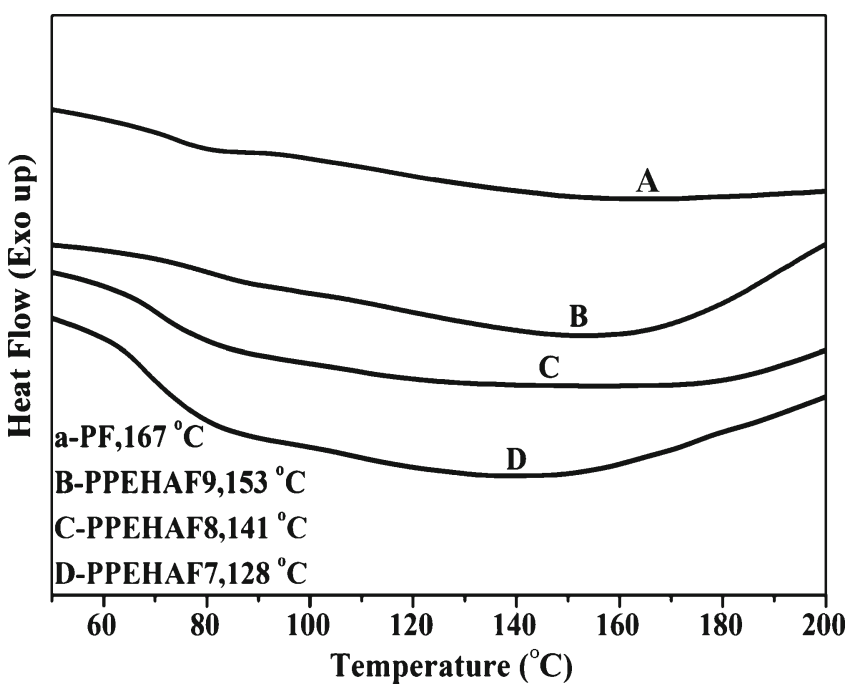

Figure 2. DSC thermograms of PF and PF/PEHA full IPNs at heating rate of $10^{\circ} \mathrm{C} / \mathrm{min}$. 
Table 1. $M_{\mathrm{c}}$ of Novolac-PEHA IPNs derived from Hourston equation.

\begin{tabular}{lclcc}
\hline & Composition & \multicolumn{2}{c}{$M_{\mathrm{c}}\left(\times 10^{-2}\right)$} \\
\cline { 3 - 4 } Sample & PF:PEHA(w/w) & Semi IPN & Full IPN \\
\hline PF & $100 / 0$ & & 4.02 & \\
PPEHAS9 & $90 / 10$ & 5.001 & & \\
PPEHAS8 & $80 / 20$ & 6 & 4.698 \\
PPEHAS7 & $70 / 30$ & 7.092 & 5.492 \\
PPEHAF9 & $90 / 10$ & & 6.82 \\
PPEHAF8 & $80 / 20$ & & \\
PPEHAF7 & $70 / 30$ & & \\
\hline
\end{tabular}
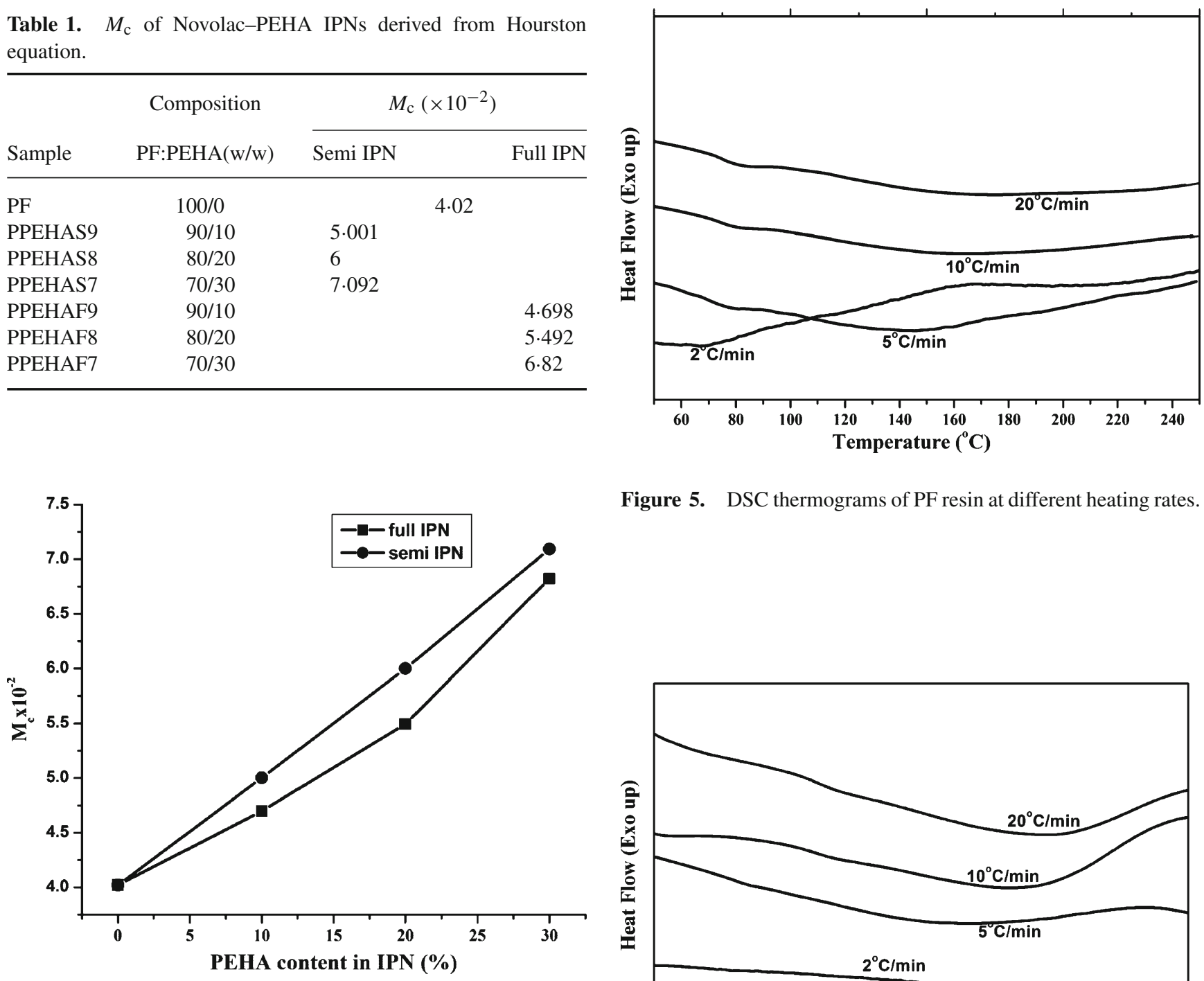

Figure 3. Variation of $M_{\mathrm{c}}$ with PEHA content (\%) in PF/PEHA IPNs.

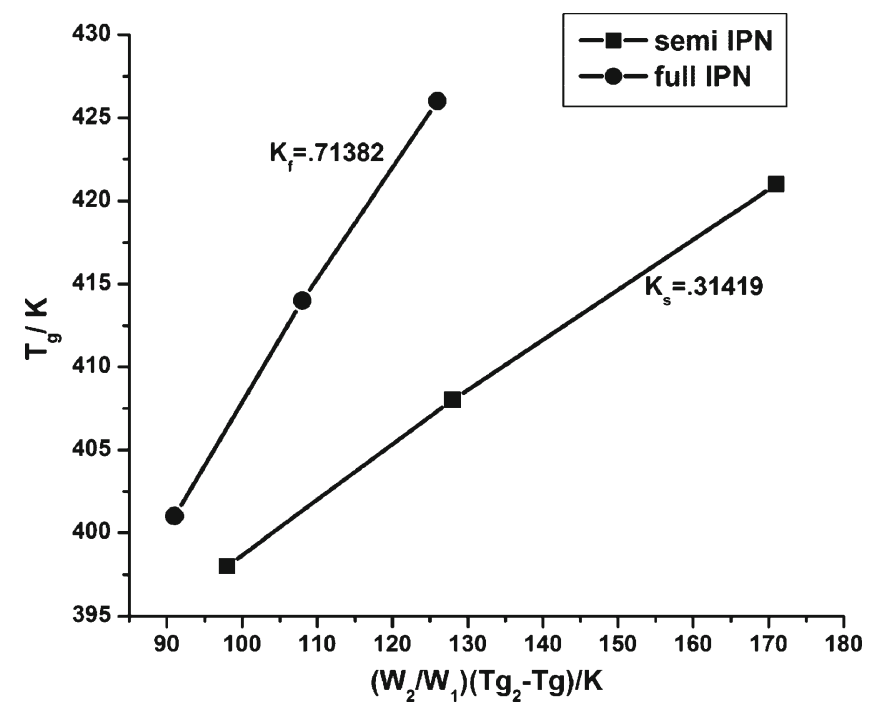

Figure 5. DSC thermograms of PF resin at different heating rates.
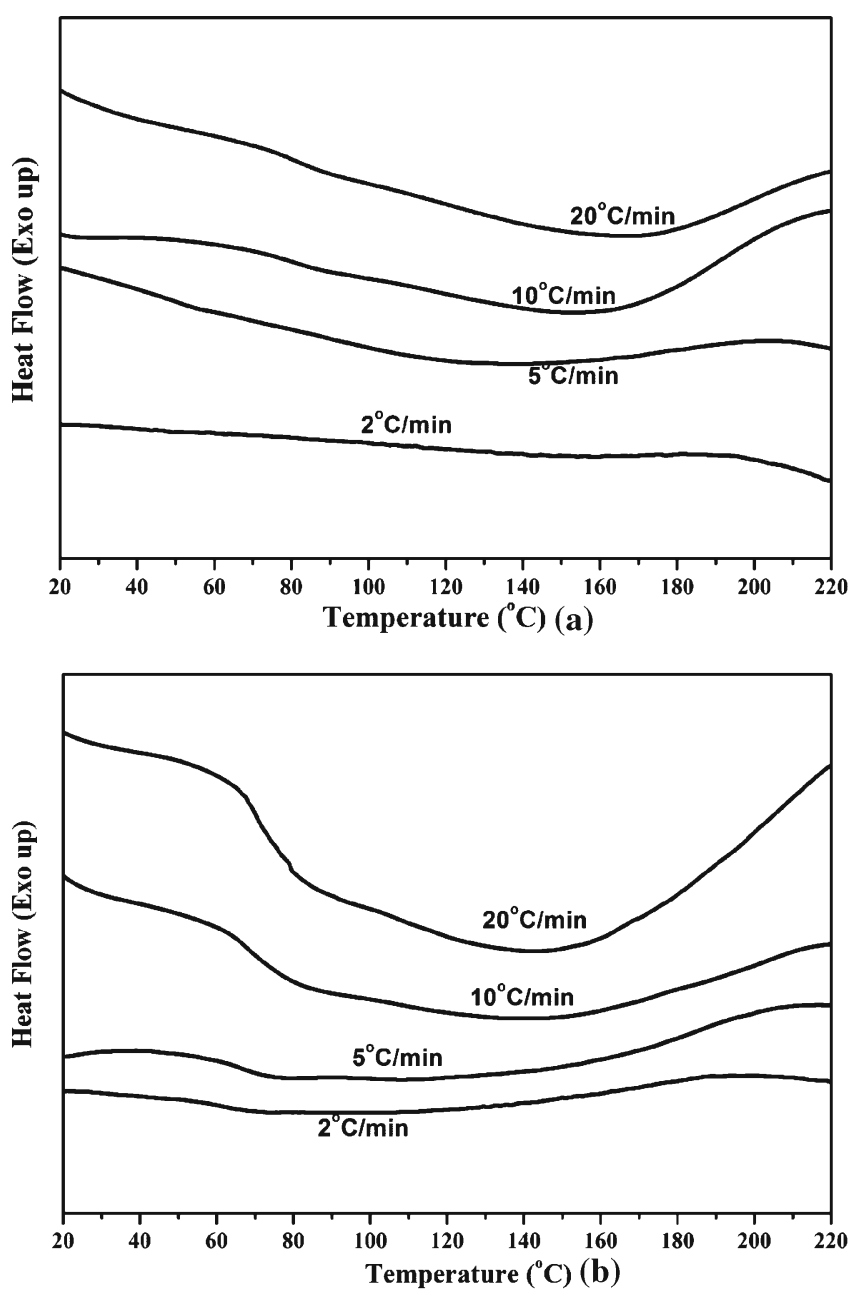

Figure 6. DSC thermograms of PF/PEHA full IPNs a.

Figure 4. Plot of Gordon-Taylor equation for PF/PEHA IPNs. 

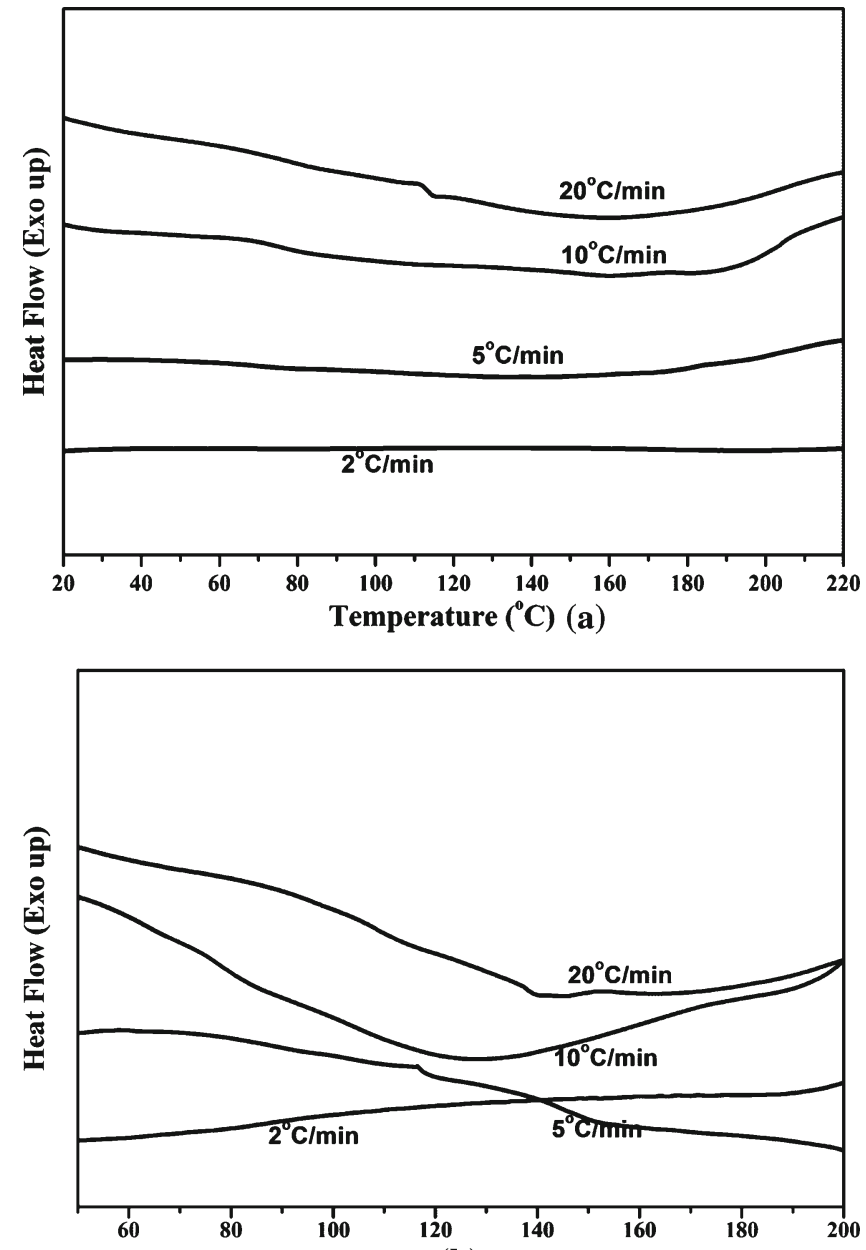

(b)

Figure 7. DSC thermograms of PF/PEHA semi IPNs a. (90:10 w/w) and b. (70:30 w/w) at different heating rates.

according to conventional method (Chang et al 2004). Similar result is found in figure 2, for full IPNs, except the span of glass transition region. The narrower width of transition for full IPNs than that in case of the semi IPNs may be because of the better phase mixing due to formation of more compact domains of crosslinked acrylate as well as inclusion of homopolymer chains of the comonomer crosslinker in between the phenolic and acrylic network. The distinct single transition point in all the cases support compatibility of the two components involved.

According to Jiang et al (1994) method, the average molecular weight $\left(M_{\mathrm{c}}\right)$ between two linkages for a crosslinked polymer is given by

$$
T_{\mathrm{g}}-T_{\mathrm{g}, 0}=\frac{3.9 \times 10^{4}}{M_{\mathrm{c}}},
$$

where $T_{\mathrm{g}}$ is the glass transition temperature of IPN and $T_{\mathrm{g}, 0}$ the glass transition of the uncured $\mathrm{PF}\left(70^{\circ} \mathrm{C}\right)($ Chen-Chi et al 1997).
Using (1), $M_{\mathrm{c}}$ values (table 1) for IPNs were calculated and plotted against PEHA content in IPNs as shown in figure 3. Higher the value of $M_{\mathrm{c}}$ lower is the crosslink density. Pure phenolic resin possesses higher degree of crosslink density compared to that of the IPNs. Full IPNs possess higher degree of crosslinking than that of corresponding

Table 2. $E_{\mathrm{a}^{\prime}}$ for glass transition obtained from DSC thermograms of Novolac/PEHA IPN.

\begin{tabular}{lcccc}
\hline & Composition & \multicolumn{2}{c}{$E_{\mathrm{a}}(\mathrm{kJ} / \mathrm{mol})$} \\
\cline { 3 - 4 } Sample & PF:PEHA(w/w) & Semi IPN & Full IPN \\
\hline PF & $100 / 0$ & & 77.69 \\
PPEHAS9 & $90 / 10$ & 51.03 & & \\
PPEHAS8 & $80 / 20$ & $48 \cdot 22$ & \\
PPEHAS7 & $70 / 30$ & 44.06 & 71.25 \\
PPEHAF9 & $90 / 10$ & & $69 \cdot 00$ \\
PPEHAF8 & $80 / 20$ & & 68.20 \\
PPEHAF7 & $70 / 30$ & & \\
\hline
\end{tabular}
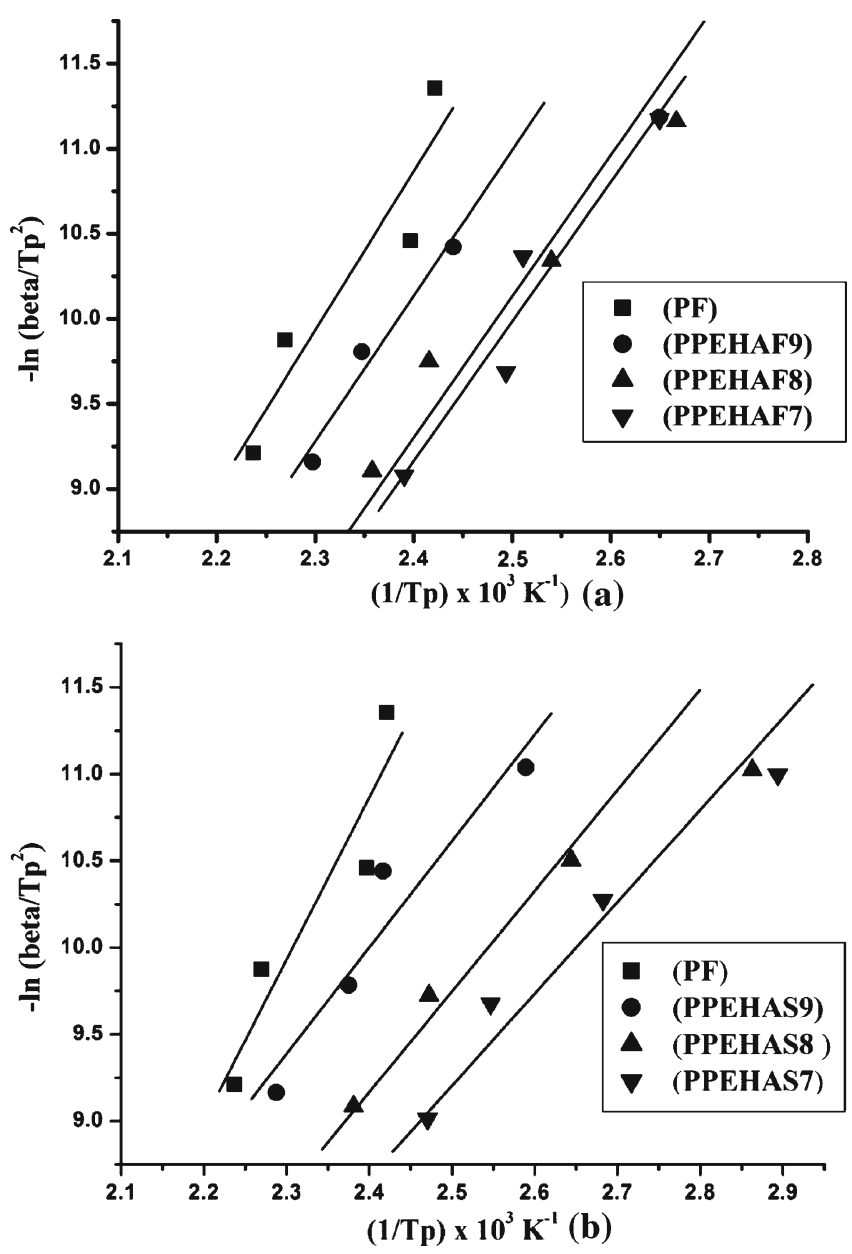

Figure 8. Plot of $-\ln \left(\beta / T_{\mathrm{p}}^{2}\right)$ vs $1 /\left(T_{\mathrm{p}}\right) \times 10^{3} \mathrm{~K}^{-1}$ for PF/PEHA a. for full IPNs and $\mathbf{b}$. for semi IPNs. 
semi IPNs. This may be explained on the basis of shielding effect, exerted by the high molecular weight PEHA chains formed in situ, surrounding the reactive methylol groups on novolac chains, thereby reducing possibility of crosslinking of the latter in the IPNs. However, in full IPNs, crosslinked and compact domains of PEHA, are less capable of interacting with the methylol groups in a similar fashion as in semi IPNs allowing crosslinking of the novolac chains to a higher extent. Fox and co-workers applied Gordon-Taylor equation (Lin and Lee 1995) to estimate the intermolecular interaction in polymer blends:

$$
T_{\mathrm{g}}=T_{\mathrm{g} 1}+K\left(\frac{W 2}{W 1}\right)\left(T_{\mathrm{g} 2}-T_{\mathrm{g}}\right),
$$

where $W_{1}$ and $W_{2}$ are the weight fractions of acrylate and novolac in the IPN compositions, $T_{\mathrm{g} 1}$ and $T_{\mathrm{g} 2}$ are the glass transitions for acrylate and phenolic network, respectively and $K$ the adjustable parameter, which increases with the extent of interaction between components. A plot of Gordon-Taylor equation is given in figure 4. The measured $K$ values ( $K_{\mathrm{f}}$ for full IPNs and $K_{\mathrm{s}}$ for semi IPNs, respectively) suggest that full IPNs have stronger intermolecular interaction than semi IPNs. This may be due to the incorporation of co-monomer crosslinker in the former which acts as better compatibilizer too between the two phases. Figure 5 shows DSC thermograms of pure PF at different heating rates. Figures $6 \mathrm{a}-7 \mathrm{~b}$ show variation in the endothermic peak temperature $\left(T_{\mathrm{p}}\right)$ with the variation in heating rate
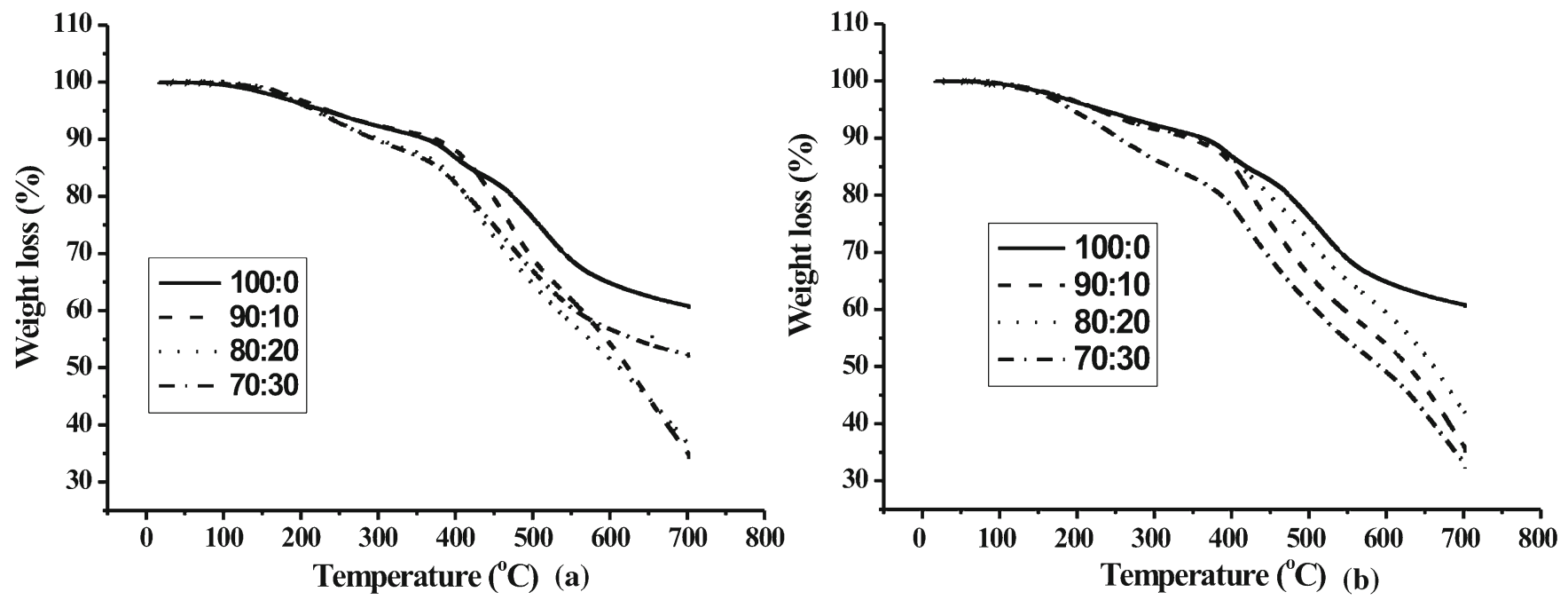

Figure 9. TGA thermograms of PF/PEHA (a) full and (b) semi IPNs.

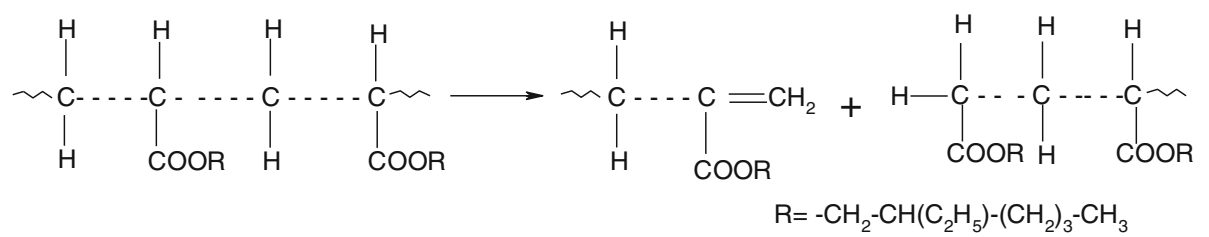

Scheme 1. Degradation of polyacrylate in absence of phenolic resin.

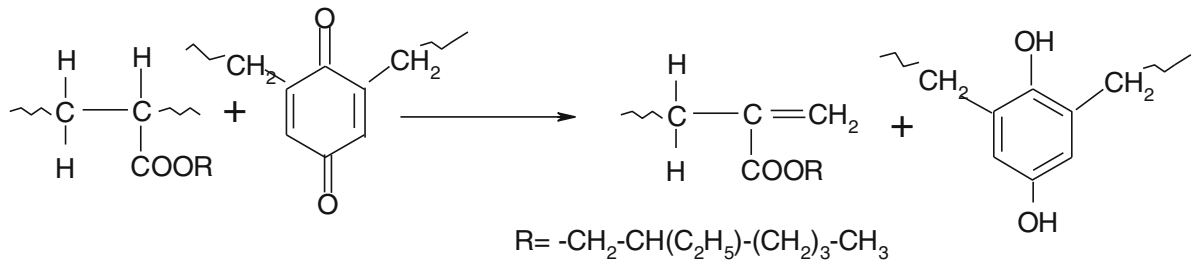

Scheme 2. Degradation of polyacrylate in presence of phenolic resin. 


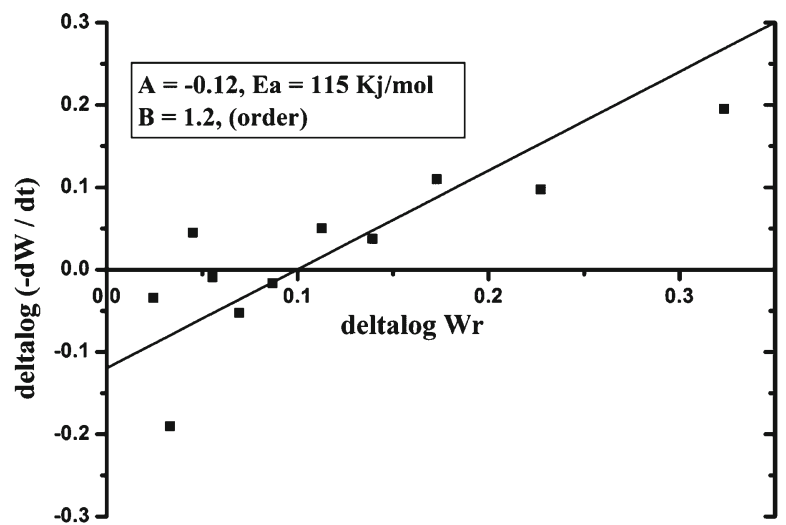

(a)
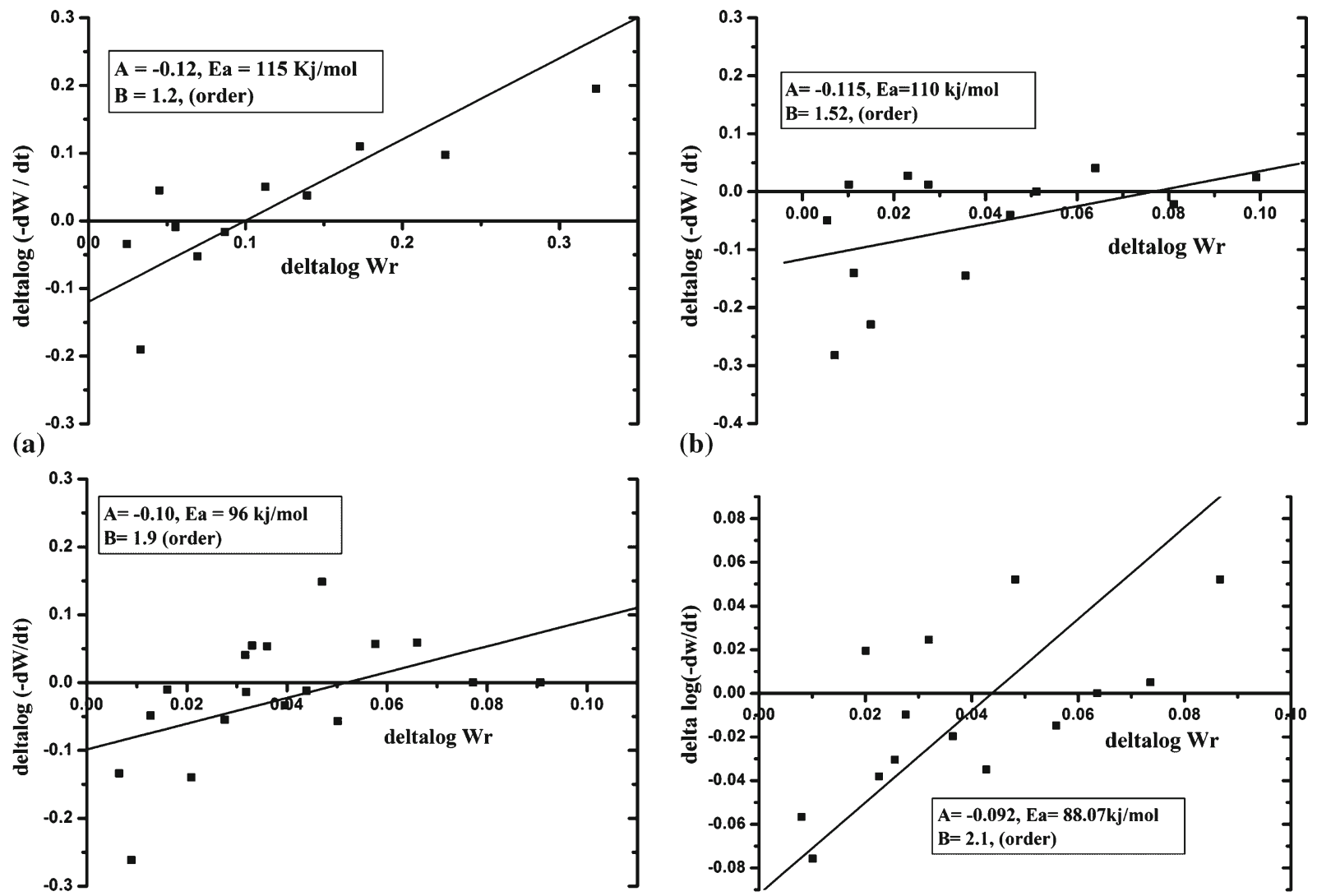

(b)

(c)

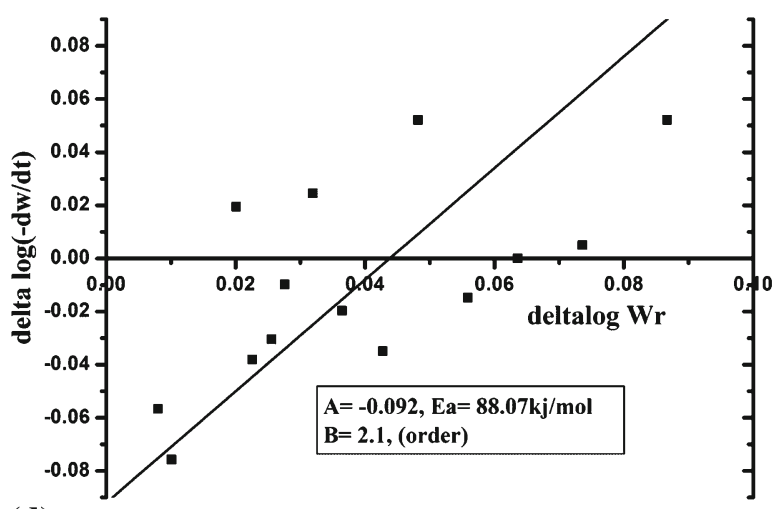

(d)

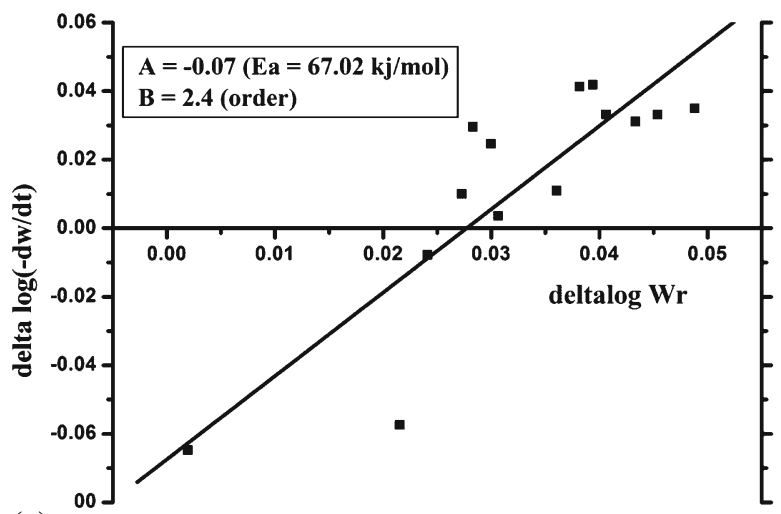

(e)

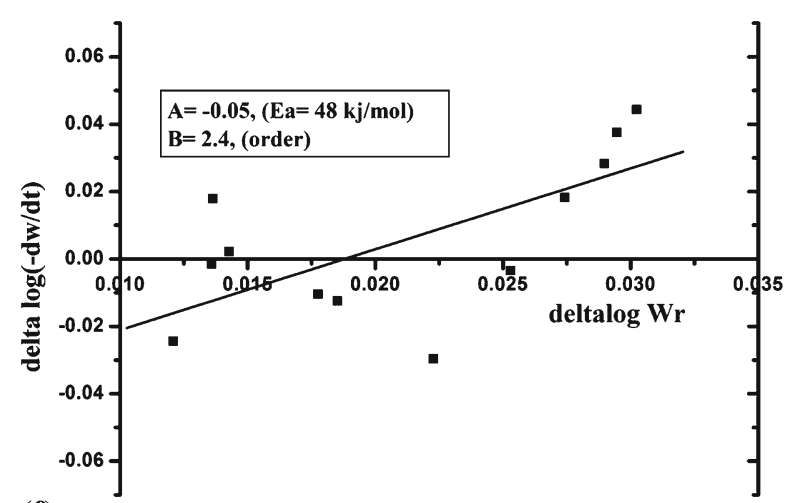

(f)

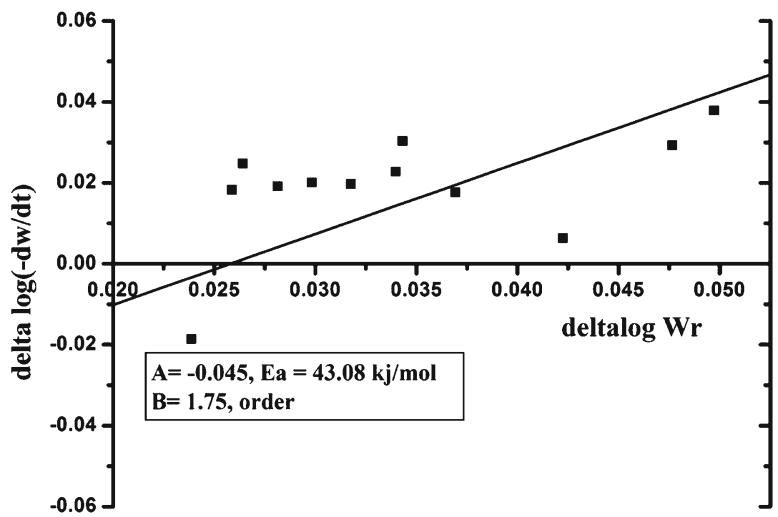

(g)

Figure 10. Plot of $\Delta \log (-\mathrm{d} W / \mathrm{d} t)$ vs $\Delta \log W_{\mathrm{r}}$ a. for pure PF, b. for PF/PEHA full IPN(90:10) w/w, c. for PF/PEHA full IPN(80:20)w/w, d. for PF/PEHA full IPN(70:30)w/w, e. for PF/PEHA semi IPN(90:10) w/w, f. for PF/PEHA semi $\operatorname{IPN}(80: 20) \mathrm{w} / \mathrm{w}$ and $\mathbf{g}$. for PF/PEHA semi IPN(70:30) w/w. 
$\left(\beta,{ }^{\circ} \mathrm{C} \min ^{-1}\right)$ for PF/PEHA full and semi IPNs. Higher the heating rate higher is the $T_{\mathrm{p}}$. On the basis of the linear relation between peak temperature $\left(T_{\mathrm{p}}\right)$ and heating rate $(\beta)$, Kissinger (Kissinger 1957; Chunhua et al 2010) proposed the following (3) to determine the kinetic parameter e.g. activation energy, $E_{\mathrm{a}^{\prime}}\left(\mathrm{kJ} \mathrm{mol}^{-1}\right)$ of the corresponding transition in DSC thermogram:

$$
-\ln \left(\beta / T_{\mathrm{p}}^{2}\right)=\left(E_{\mathrm{a}^{\prime}} / R\right)\left(\frac{1}{T_{\mathrm{p}}}\right)-\ln \left(\frac{A R}{E_{\mathrm{a}^{\prime}}}\right),
$$

where $R=8.3143 \mathrm{~J} \mathrm{~mol}^{-1} \mathrm{~K}^{-1}$ and $A$ the pre-exponential factor $\left(\mathrm{min}^{-1}\right)$.

From the slope of Kissinger plot $\left\{-\ln \left(\beta / T_{\mathrm{p}}^{2}\right)\right\}$ vs $1 /\left(T_{\mathrm{p}}\right), E_{\mathrm{a}}$ is calculated as shown in table 2 . Figures $8 \mathrm{a}$ and $\mathrm{b}$ show the Kissinger plot for full IPNs and semi-IPNs, respectively. IPNs are showing lower $E_{\mathrm{a}^{\prime}}$ for the endothermic transition than the pure phenolic resin itself and it may be because of reduced crosslink density in the former. Also it is very apparent that full IPNs, having both the polymeric networks crosslinked, possess greater intermolecular interaction and requires higher activation energy for glass transition; hence higher $T_{\mathrm{g}}$ of the latter samples compared to that of the semi IPNs. Furthermore, with the decrease of novolacPEHA blend ratio decrease in the values of $E_{\mathrm{a}^{\prime}}$, for both full and semi IPNs, is observed. This is due to the increase in $M_{\mathrm{c}}$ found in the samples facilitating the segmental mobility of the component polymers.

\subsection{Thermogravimetric analysis (TGA)}

Thermograms of pure phenol-fromaldehyde and its IPNs with PEHA show (figures 9a and b) two step degradation. However, the percent residue left of pure phenolic resin is found to be higher compared to that of IPNs. Semi IPNs show poorer stability compared to that of full IPNs with corresponding composition. The probable reaction mechanism of such a degradation of phenolic resin in presence and absence of the acrylic network interpenetration has already been reported (Madorsky 1964; Chakrabarty and Goswami 2006) as shown in schemes 1 and 2. Estimation of activation energy and order of reaction is done here to ensure mechanism of reaction further.

In the present work, differential method given by Freeman and Carroll (Gurnule and Katkamwar 2010; Masram 2009) for determining rate equation parameters, viz. activation energy $\left(E_{\mathrm{a}}\right)$ and order of reaction $(n)$, has been utilized. The equation can be written as:

$$
\begin{aligned}
\Delta 2.303 \log (-\mathrm{d} w / \mathrm{d} t)= & n 2.303 \log W_{\mathrm{r}} \\
& -\left(E_{\mathrm{a}} / R\right) \Delta(1 / T),
\end{aligned}
$$

where $W_{\mathrm{r}}$ is the weight fraction remaining in a TGA run at a constant heating rate, $\mathrm{d} W / \mathrm{d} t$ the rate of weight loss which is equivalent to the rate of reaction, determined from the derivative curve of TGA at a particular temperature, $T$. Plot of $\Delta \log (-\mathrm{d} W / \mathrm{d} t)$ vs $\Delta \log W_{\mathrm{r}}$ (figures $10 \mathrm{a}-\mathrm{g}$ ) gives $n$ and $E_{\mathrm{a}}$ as shown in table 3 .
Table 3. Kinetic parameters derived by using Freeman-Carroll and Flynn-Wall method (from $400-700^{\circ} \mathrm{C}$, at $10^{\circ} \mathrm{C} / \mathrm{min}$ ).

\begin{tabular}{lcc}
\hline Sample & $\begin{array}{c}\text { Order of } \\
\text { degradation }(n)\end{array}$ & $\begin{array}{c}\text { Activation energy, } E_{\mathrm{a}}(\mathrm{kJ} / \mathrm{mol}) \\
\text { Freeman-Carroll }\end{array}$ \\
\hline PF & 1.2 & 115 \\
PPEHAF9 & 1.52 & 110 \\
PPEHAF8 & 1.9 & 96 \\
PPEHAF7 & 2.1 & 88 \\
PPEHAS9 & 2.4 & 67 \\
PPEHAS8 & 2.4 & 48 \\
PPEHAS7 & 1.75 & 43 \\
\hline
\end{tabular}

First order decomposition of phenolic resin ensures involvement of only one reactant molecule in the rate determining step as is proposed in literature (Chakrabarty and Goswami 2006). On the other hand, second order kinetics of decomposition of phenolic-acrylic IPNs indicates involvement of two independent reactant molecules in the second step of two-stage degradation, one being phenolformaldehyde, the other must be PEHA. With increase in acrylic percentage in IPNs, the order of reaction remained the same while the activation energy decreased gradually indicating the presence of PEHA molecules facilitating the decomposition of phenolic resin.

\section{Conclusions}

Pure PF possesses higher crosslink density than that of IPNs. Also crosslink density of full IPNs is found to be higher compared to that of semi IPNs of corresponding composition. Lower the crosslinking density greater is the toughening of the system. This effect predominates further due to incorporation of more acrylate in the blend. Stronger interaction between the components in full IPNs allows better mixing. The effect of such interaction is clearly opposing the effect of greater degree of crosslinking in the full IPNs; hence the narrower DSC endotherm and marginally higher $T_{\mathrm{g}}$ values were observed for full IPNs compared to that of corresponding semi-type. $E_{\mathrm{a}^{\prime}}$ of glass transition for PF and PF/PEHA IPNs were evaluated applying Kissinger equation. Higher the acrylic content in the IPNs lower is the activation energy for corresponding transition. Also $E_{\mathrm{a}^{\prime}}$ for IPNs are seen lower than that of PF. Full IPNs possess higher activation energy compared to that of semi IPNs which may be due to the

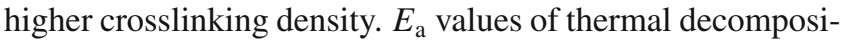
tion were investigated by TGA. First order decomposition of phenolic resin ensures involvement of only one molecule in the rate determining step and second order kinetics of decomposition of phenolic-acrylic IPNs indicates involvement of two independent molecules in the second step of two-step degradation, one being phenol-formaldehyde and the other must be PEHA. Furthermore, activation energy of IPNs 
decreased gradually with increasing PEHA content indicating the presence of PEHA molecules facilitating the decomposition of phenolic resin. Full IPNs exhibit higher activation energy for thermal decomposition compared to that of corresponding semi IPN due to greater degree of crosslinking. Thus PF/PEHA full IPN formation may be the most useful technique of toughening phenol-formaldehyde.

\section{Nomenclature list}

\begin{tabular}{|c|c|}
\hline 3 & phenol formaldehyde resin; \\
\hline PEHA, & poly (2-ethyl hexyl acrylate); \\
\hline IPN, & interpenetrating network; \\
\hline $\mathrm{SC}$, & differential scanning calorimetry; \\
\hline$M_{\mathrm{c}}$ & $\begin{array}{l}\text { average molecular weight between two } \\
\text { successive crosslinks; }\end{array}$ \\
\hline $\begin{array}{l}W_{1}, W_{2}, \\
E_{\mathrm{a}^{\prime}},\end{array}$ & $\begin{array}{l}\text { weight fractions of PEHA and PF in IPNs; } \\
\text { activation energy for glass transition } \\
\text { in DSC curve; }\end{array}$ \\
\hline$\beta$, & rate of heating; \\
\hline TGA & thermogravimetric analysis; \\
\hline & $\begin{array}{l}\text { activation energy of thermal decomposition } \\
\text { in TGA curve; }\end{array}$ \\
\hline$n$, & order of decomposition reaction; \\
\hline & peak temperature in the DSC thermograms; \\
\hline$W_{\mathrm{r}}$, & $\begin{array}{l}\text { weight fraction remaining in a TGA run } \\
\text { at constant heating rate; }\end{array}$ \\
\hline
\end{tabular}

\section{References}

Bandyopadhyay D, Chakrabarty D, Mandal P K and Goswami S 2003 J. Appl. Polym. Sci. 90412

Chakrabarty D and Goswami S 2006 J. Appl. Polym. Sci. 992857

Chakrabarty D, Goswami S and Nad S 2005 J. Appl. Polym. Sci. 97 2407

Chang Y J, Inagaki M J, Kim Y J, Kim M, Park C R and Yun C H 2004 Colloid Interf. Sci. 274552

Chen-Chi M, Chu Peter P, Chiang, Hew-Der W, Long Y, Lee Y, Fu S and Wang L Y 1997 Full Nano Carbon Nanostr. 5935

Chunhua L, Dongna Z and Shuhua Q 2010 J. Appl. Polym. Sci. 115 3675

Gardziella A, Knop A and Pilato L A 2000 Phenolic resins: Chemistry, applications, standardization, safety and ecology (Germany: Springer) 2nd ed.

Gurnule W B and Katkamwar S S 2010 E-J. Chem. 71380

Hwang T S, Jonathan M R, Kim D J, Kim H Z and Lee Y K 2003 J. Appl. Polym. Sci. 892589

Jiang M, Xiao H and Yu T 1994 Polymer 355529

Kissinger H E 1957 Anal. Chem. 291702

Knop A and Scheib W 1979 Chemistry and application of phenolic resin (New York: Springer-Verlag)

Lin Mu-Shih and Lee Shin-Tien 1995 Polymer 364561

Madorsky S L 1964 Polymer reviews (New York: Interscience) 289

Masram D T 2009 E-J. Chem. 6830

Papa A J and Sunshine N B 1973 Flame retardancy of polymeric material (New York: Marcel Dekker Inc.) Vol. 2

Wang H, Yan Y, Yu Y, Zhao T and Zhi L 2002 Macromol. Rapid Commun. 2344

Wolfgang H 2002 'Phenolic resins' in Ullmann's encyclopedia of industrial chemistry (Weinheim: Wiley-VCH) 